\title{
Heterogeneity Aspects of Breast for Breast Cancer Patients Change due to COVID-19 ${ }^{1}$ Shawni Dutta and ${ }^{2}$ Prof. Samir Kumar Bandyopadhyay \\ ${ }^{1}$ Department of Computer Science, The Bhawanipur Education Society College, Kolkata, India. \\ ${ }^{2}$ Academic Advisor, The Bhawanipur Education Society College, Kolkata, India.
}

\begin{abstract}
Breast cancer develops from cells lining the milk ducts and slowly grows into a lump or a tumour. Breast cancer may be invasive or non-invasive. Invasive cancer spreads from the milk duct or lobule to other tissues in the breast, whereas, non-invasive ones lack the ability to invade other breast tissues. Non-invasive breast cancer is called in situ and may remain inactive for entire lifetime. Due to heterogeneity nature of breast, density as well as masses is variable in size and shape. A dataset of 18056 patients are collected from 20 Government Hospitals and 50 Private Hospitals in West Bengal before COVID-19 and after COVID-19. The classification of patients are made on three classes- Normal, Sign of Abnormality and Abnormality. The reports of MRIs of patients in January 2020 and February 2020 are collected from different hospitals. It is treated as dataset before COVID-19. MRIS of patients in April 2020 and May 2020 are dataset during COVID-19. The entire datasets are accumulated for testing of any change in patients MRIS after the official announcement of new virus COVID-19 in March 2020. The aim of the paper is to make a comparison of any change in size and shape of masses of MRIs of patients before and after COVId-19. All collected MRIs reports are diagnosed by radiologists of hospitals.
\end{abstract}

\section{Keywords: COVID-19, Breast Cancer, Breast Cancer (Suspected), Mammogram Images and Invasive Cancer}

\section{Introduction}

Breast is referred as mammary gland in scientific term. With reference to the Merriam-Webster dictionary the Breast is either of the pair of mammary glands extending from the front of the chest in pubescent and adult females of humans and some other mammals. It is also either of the analogous but rudimentary organs of the male chest especially when enlarged [1].

Several researches have shown that study of heterogeneity in mammogram may save lives and increases treatment options [2]. Breast cancer, a malignant tumour developed from breast cells is considered to be one of the major causes for the increase in mortality among women, especially in developed and developing countries [3]. 
Most masses in breast are benign and do not grow uncontrollably or spread. Some breast cancers are called in situ because they are confined within the ducts (ductal carcinoma in situ) or lobules (lobular carcinoma in situ) of the breast. Nearly all cancers at this stage can be cured. Lobular carcinoma in situ (also known as lobular neoplasia) is an indicator of increased risk for developing invasive cancer in either breast.

The breast cancer diagnosis process during COVID-19 needs attention whether shape of the abnormal masses is changed or not. If there is any change then it requires immediate attention for treatment.

It was mentioned in the abstract that a study over 50 patients has been made to identify any changes before or after COVID-19. This paper detects change of masses and their shapes.

\section{Related Works}

A search on internet has been made relating to such study but nothing is found. So the proposed method for observing the changes in mass in breast is compared with the existing methods for detection of breast cancer.

Researchers proposed Computer Aided Diagnosis (CAD) for detection of abnormal masses [4]. The recent studies show that the majority of clinical tests including digital mammography and biopsies on patients performed manually. So it is wastage of valuable time of medical practitioners, if it is found benign. At the same time the possibility of false detection is increasing in the manual system. The efficiency of CAD system can screen the benign cases easily and assist the experts in terms of qualitative and quantitative precision. Thus CAD has become a part of routine clinical detection of breast cancer on mammograms at many mass screening program and hospitals [5].

CAD system starts from image preparation, pre-processing, features extraction, registration, segmentation and detection of abnormal mass in breast, if present. The process of segmentation of the part of the affected breast is most important. Segmentation is related with the pectoral muscle suppression, edge detection, determination of Region of Interest (ROI), anatomical segmentation, segmentation of abnormalities like mass and micro-calcification.

The preparation, pre-processing and features extraction are normally made for making the image in such a way that no unwanted parts are seen in the images which are the input to next steps to detect correctly the abnormalities in the next phases.

Researchers reviewed several well accepted mammogram image registration techniques and classified them according to their functionality. The primary objective of registration is to align one image to another by finding the optimal transformation or mapping function. It involves a searching plan to enhance a similarity measure. This similarity measure is determined using 
certain characteristics of the images. Feature space, transformation, similarity measure and search strategy constitute a typical registration framework in automated image processing systems like CAD. The enhancement of mammogram is also performed by intensity and contrast manipulation, noise reduction, background removal, edge sharpening, filtering etc.

\section{Data Set}

Dataset is a collection of similar and related data stored for processing. Further this can be defined as a collection of data that contains individual data units organized in a specific format. A data set generally contains a collection of many types of data. Medical dataset can be defined as a collection of pieces of information, especially those that are part of a collection to be used for the the diagnosis of diseases. In this paper data set, containing mammogram images from hospitals along with their reports, are stored for the same selected patients before and after COVID-19. The shapes of masses of patients before COVID-19 are analyzed since these data are compared with the shapes of masses of patients after COVID-19. These data are obtained from different Government hospitals as well as from Private Hospitals. Generally, data set before COVID-19 are normally stored in the hospitals along with details of patient information as well as their diagnostic reports. The radiologist has provided all relevant information regarding any abnormality present. The images are classified into normal, tends to be sign of breast cancer and also patients who already detected as breast cancer patients. These detections are based on the diagnosis of radiologists. The mass can also be classified according to the type of mass present such as well-defined/circumscribed masses, spiculated masses, ill- defined masses etc. In this paper the main consideration is to detect any change of shape in masses and also normal/ tends to breast cancer patients may have sign of abnormal mass in their MRI.

\section{Proposed Method}

The objective of the paper is to find out the presence of abnormal mass created in normal patients and as well as in tends to breast cancer patients. Also if there is any change of masses in confirmed breast cancer patients due to COVID-19 then it has to be specified for immediate treatment.

The masses i.e. tissues which are absent in normal breast anatomy is called abnormal masses. The size and shape of density of masses are variable in nature. So it has a unique impression in the mammogram image. It is the role of medical practitioners to draw a conclusion about the nature of abnormalities. So the proposed method is described briefly in the next paragraph.

Digital Mammograms initially pre-processed to improve the quality of the image. Detection of abnormalities is based on the images obtained after segmentation. Anatomical Segmentation of Breast Region of Interest (ROI) determines abnormalities in breast. It not only segregated the 
regions but also removes all unwanted dots, edges and discrete objects from the edge map. Segmentation process is made on the edge map to differentiate various regions on the breast based on their intensity values. Actually each region has a different intensity value. Different intensity values of fatty tissues, glands, lobules and the ducts are segregated into different regions. Mass, tumours or calcifications present within the breast has distinctly higher intensity values than the normal tissues of the breast. All the closed structures on basis of their intensity values are categorized. Pixels intensities are also varied within each segmented region but the majority of the pixels have similar intensity values. The arithmetic Mode value is calculated for each region from the original mammogram and replaces those pixels in the region with the computed mode values. The scanning process starts from the first row of the image and proceeds to row major order up to last row. As soon as region with higher intensity is found, coloring process started for the region by first comparing the pixel intensity at the level up to the pixel of original mammogram image. Four boundaries of each pixel located north, east, west and south are checked to find out whether they are colored or they form the boundary pixel. If the pixels are not colored as well as not boundary pixels then further searching is made. A stack is used to store the seeds, calculated from intensity value, for investigation. A List is used to store the pixels already traversed of the regions. All the pixel positions within the List are compared with the original image pixel. It then stored their intensity values for calculating the Mode value. The pixel locations of each region are replaced by the computed Mode value intensity. The proposed method enumerated each region along with their respective statistical mode value. This dataset is used for further statistical analysis. First, the Arithmetic mean for the distribution is calculated to obtain the deviations of each region. Subsequently the Standard Deviation of the dataset is calculated. Now the mean value is calculated along with the standard deviation using the dataset. Now, the regions are categorised into four discrete levels according to their colour intensity. The method highlighted the abnormal regions by Red colour and suspected region with Blue colour. The other regions are also coloured with different shades according to the different categories. Thus abnormal mass presence in the MRI of the patient is indicated with red colour. This method applied to images collected before and after COVID19. Figure 1 shows the result of a MRI of a patient (tends to be a breast cancer patient) after using the approach from the set of MRIs images of patients before COVID-19. It is shown in figure 1 . 


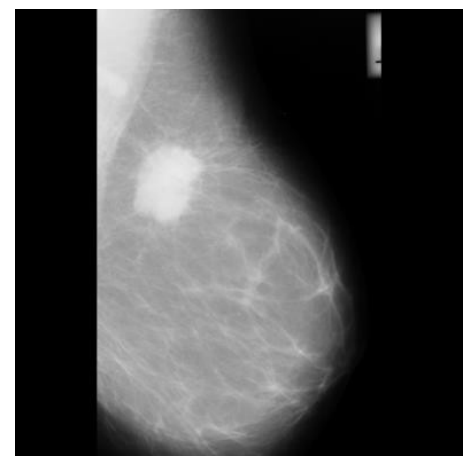

(a)

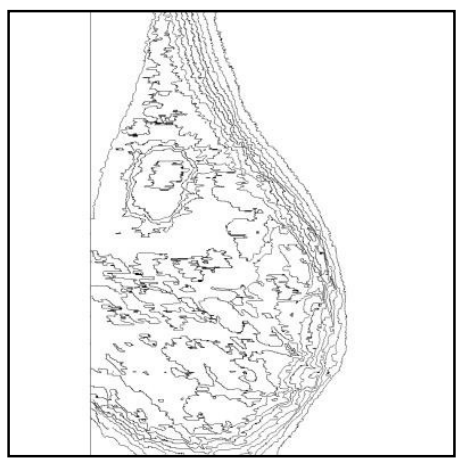

(b)

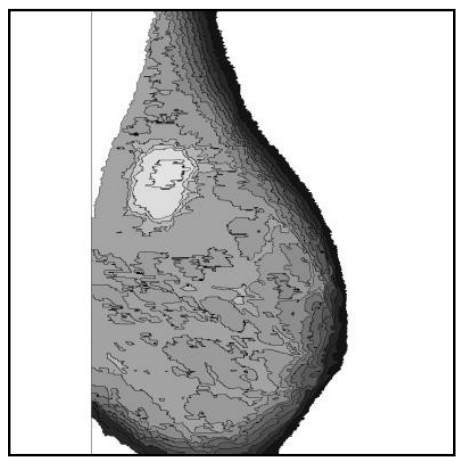

(c)

Figure 1. (a) Original Mammogram, (b) Anatomical Regions and (c) Mammogram with mass but not abnormal mass before COVID-19

MRI of same patient is analysed after COVID-19 and it indicates a creation of mass, coloured with red, after taking MRI of the patient. It is shown in figure 2.

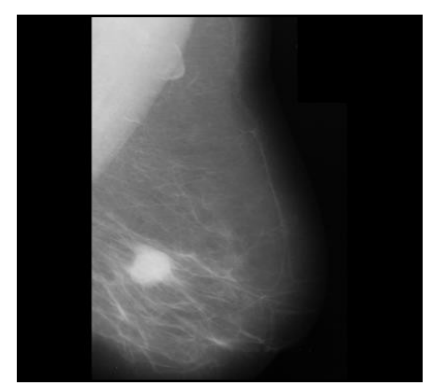

(a)

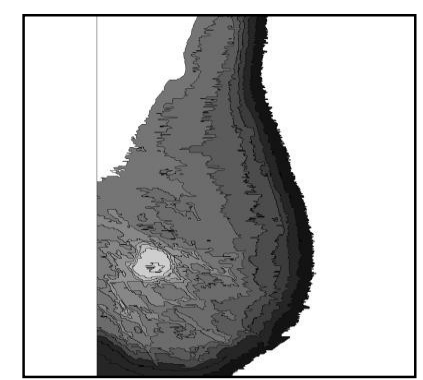

(b)

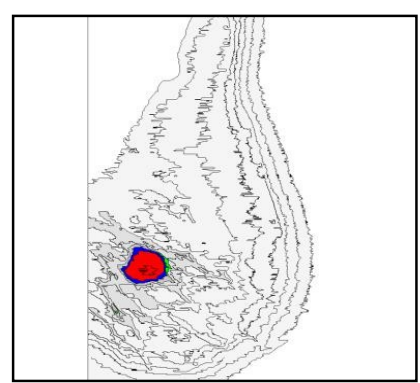

(c)

Figure 2. (a) Original Mammogram, (b) Anatomical Regions for fatty tissue and (c) Mammogram showing Abnormal Region(s) after COVID-19 with red colour

The proposed approach is now applied on Breast Cancer patient before COVID-19 and it indicates abnormality in MRI, as shown in figure 3.

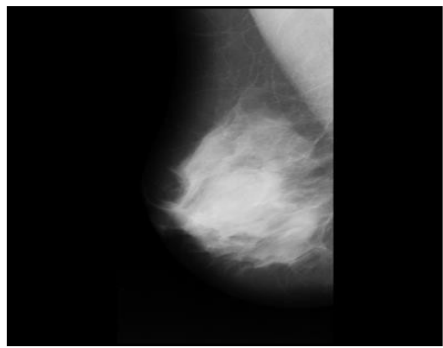

(a)

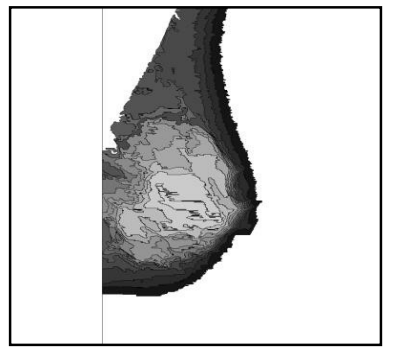

(b)

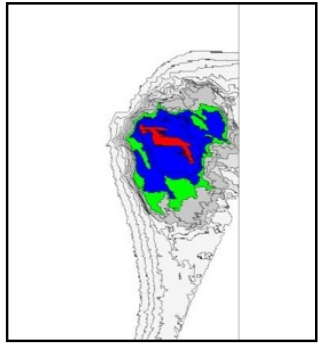

(c)

Figure 3. a) Original Mammogram, b) Anatomical Regions and c) Mammogram showing presence of abnormal region before COVID-19 
Figure 4 shows the result of MRI of same patient (in figure 3). In the output, It is shown in the output that there is presence of three abnormal masses in comparison with one abnormal mass in the mammogram of same patient after COVID-19. The parts of the increased masses are shown by red colour.

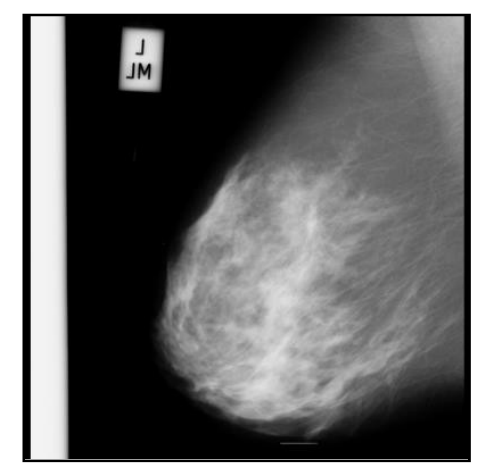

(a)

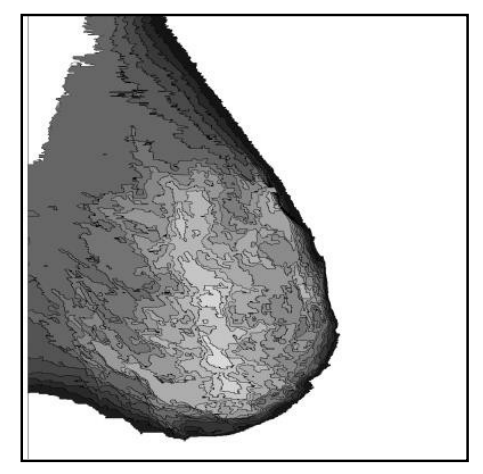

(b)

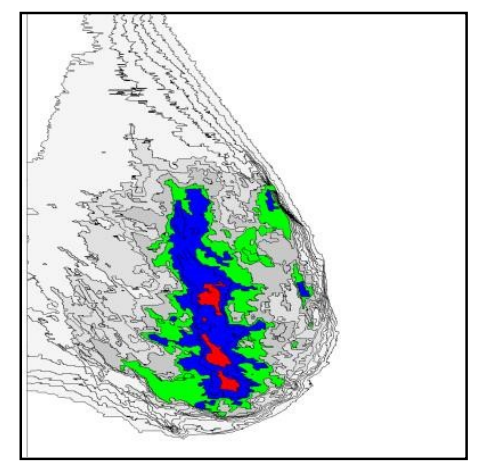

(c)

Figure 4. a) Original Mammogram, b) Anatomical Regions after proposed method and c) Mammogram showing presence of more abnormal masses after COVID-19

\section{Results and Analysis}

The proposed method applied for the entire data set before COVID-19 and during COVID-19. Initially dataset of four months of MRIs of patients along with their diagnosis by radiologists are shown in table 1- table 4. The graphical representations of diagnosis of patients for each month are given after each table in the form of figure 5 to figure 8 . The performance measure metrics such as Accuracy, F1-score, and Cohen-Kappa score are used for evaluating the prediction. Table 5 shows the performance measure of the proposed method. Figure 9 and table 6 show the collected dataset before and after COVID-19. Figure 10 to figure 12 indicate the individual measure feature for four months.

\begin{tabular}{|r|r|r|r|r|r|}
\hline Days & \multicolumn{2}{|l|}{ Total MRI Reports Collected for } & Normal & \multicolumn{2}{|l|}{ Sign of Abnormality } \\
\hline & patients came for testing breast cancer & & & \\
\hline 12 & 112 & 100 & 11 & 1 \\
\hline 16 & 80 & 67 & 7 & 6 \\
\hline 17 & 33 & 24 & 8 & 1 \\
\hline 18 & 45 & 56 & 49 & 121 \\
\hline 25 & 69 & 67 & 2 & 0 \\
\hline 26 & 77 & 70 & 4 & 3 \\
\hline 29 & 57 & 45 & 10 & 2 \\
\hline 30 & 78 & 60 & 14 & 4 \\
\hline Total & & 551 & 489 & 105 & 138 \\
\hline
\end{tabular}

Table 1 Dataset prepared for the month of January 2020 from hospitals 


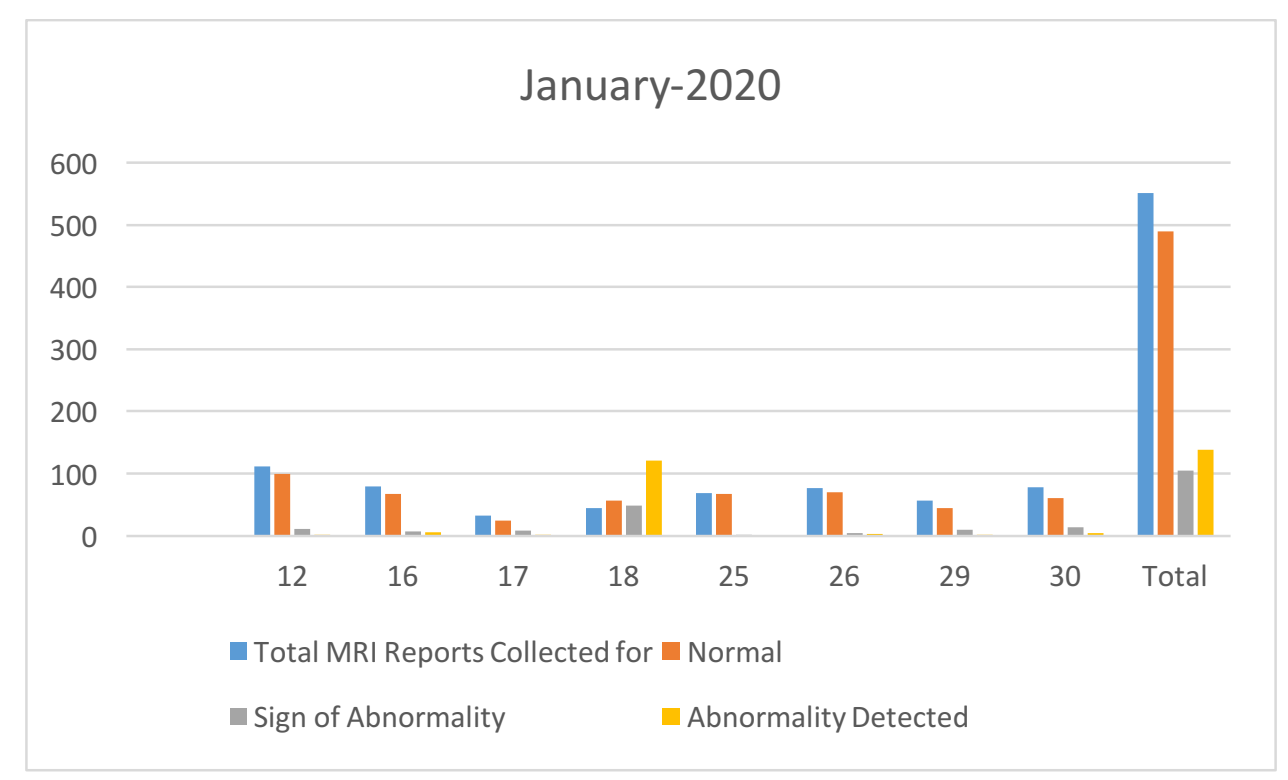

Figure 5 It indicates three types of cases and total in graphical form

\begin{tabular}{|c|c|c|c|c|}
\hline \multirow[t]{2}{*}{ Days } & \multirow{2}{*}{$\begin{array}{l}\text { Total MRI Reports Collected for patient came for testing Breast Cancer } \\
\text { patients came for testing breast cancer }\end{array}$} & \multirow[t]{2}{*}{ Normal } & \multicolumn{2}{|c|}{ Sign of Abnormality Abnormality Detected } \\
\hline & & & & \\
\hline 3 & 112 & 100 & 10 & 2 \\
\hline 10 & 200 & 185 & 10 & 5 \\
\hline 19 & 67 & 52 & 8 & 7 \\
\hline 23 & 90 & 66 & 22 & 2 \\
\hline 24 & 150 & 110 & 34 & 6 \\
\hline 25 & 70 & 50 & 12 & 8 \\
\hline 26 & 170 & 90 & 70 & 10 \\
\hline \multirow[t]{2}{*}{ Total } & 859 & 653 & 166 & 40 \\
\hline & 1718 & 1306 & 332 & 80 \\
\hline
\end{tabular}

Table 2 MRIs of various patient's data during the month of February 2020

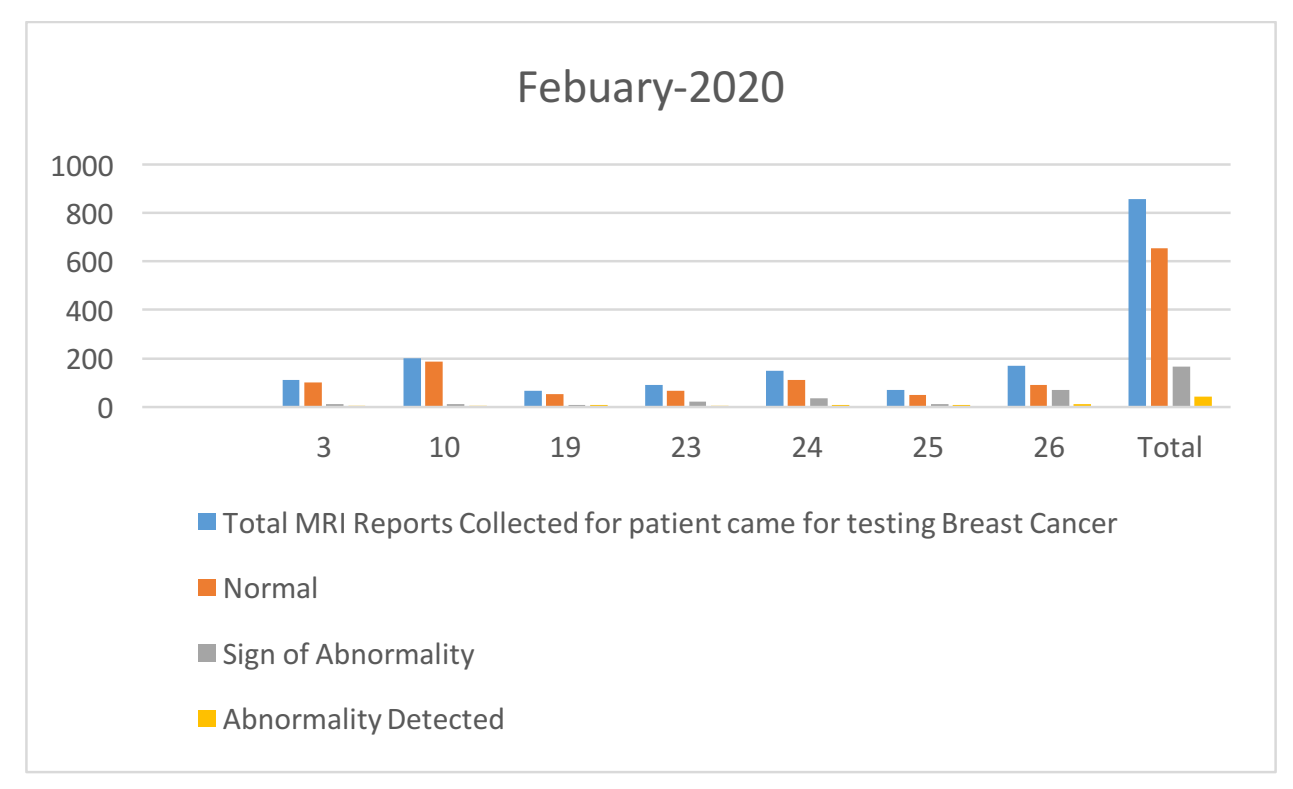

Figure 6 Abnormality detected low in comparison to January 2020 


\begin{tabular}{|c|c|c|c|c|}
\hline \multirow[t]{2}{*}{ Days } & \multirow{2}{*}{$\begin{array}{l}\text { Total MRI Reports Collected for patient came for testing Breast Cancer } \\
\text { patients came for testing breast cancer }\end{array}$} & \multirow[t]{2}{*}{ Normal } & \multicolumn{2}{|c|}{ Sign of Abnormality Abnormality Detected } \\
\hline & & & & \\
\hline 2 & 267 & 200 & 50 & 17 \\
\hline 13 & 500 & 400 & 60 & 40 \\
\hline 14 & 800 & 755 & 35 & 10 \\
\hline 15 & 30 & 28 & 1 & 1 \\
\hline 22 & 350 & 300 & 40 & 10 \\
\hline 23 & 900 & 850 & 30 & 20 \\
\hline 24 & 1200 & 1180 & 5 & 10 \\
\hline 25 & 200 & 175 & 15 & 10 \\
\hline 26 & 500 & 460 & 30 & 10 \\
\hline 27 & 1100 & 1080 & 12 & 8 \\
\hline 30 & 450 & 420 & 25 & 5 \\
\hline Total & 6297 & 5848 & 303 & 141 \\
\hline
\end{tabular}

Table 3 Dataset collected much more than before COVID-19

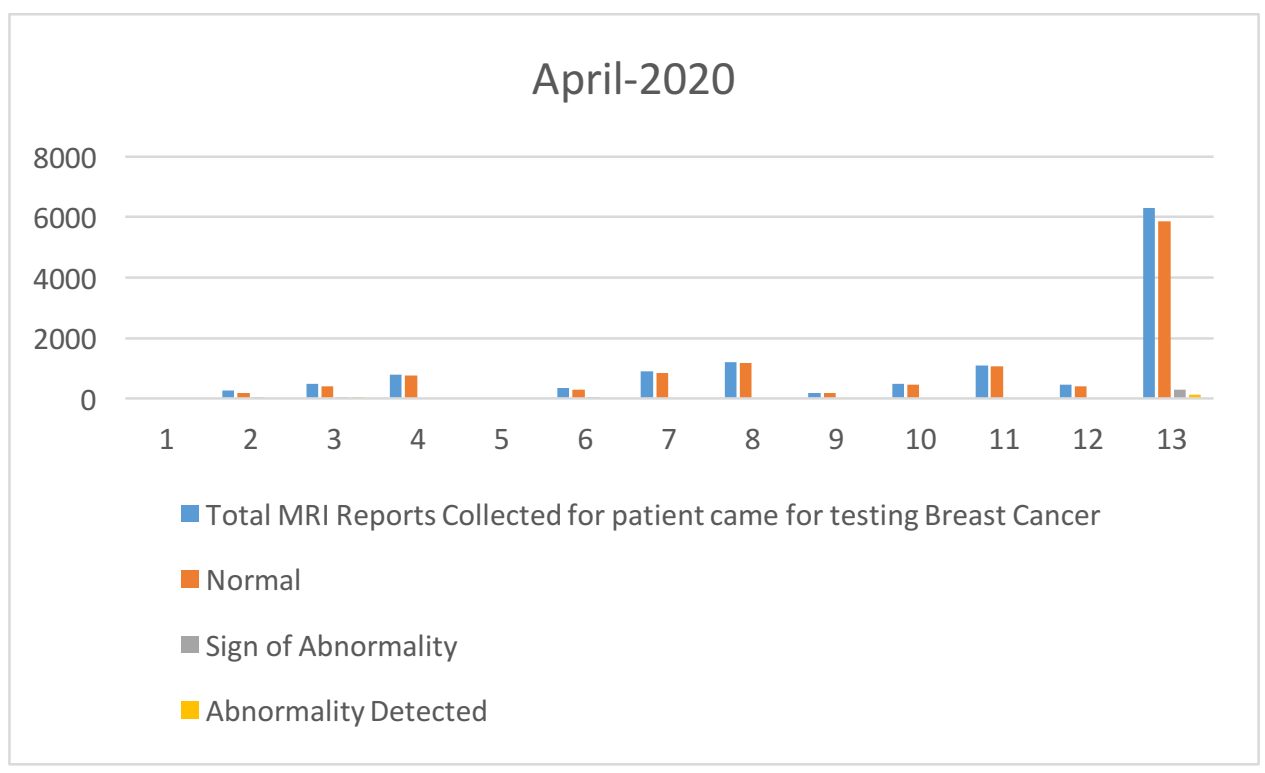

Figure 7 Tends to abnormality increased during COVID-19

\begin{tabular}{|c|c|c|c|c|}
\hline \multirow[t]{2}{*}{ Days } & \multirow{2}{*}{$\begin{array}{l}\text { Total MRI Reports Collected for patient came for testing Breast Cancer } \\
\text { patients came for testing breast cancer }\end{array}$} & \multirow[t]{2}{*}{ Normal } & \multicolumn{2}{|c|}{ Sign of Abnormality Abnormality Detected } \\
\hline & & & & \\
\hline 2 & 1500 & 1450 & 30 & 20 \\
\hline 15 & 900 & 800 & 60 & 40 \\
\hline 16 & 100 & 80 & 15 & 5 \\
\hline 17 & 870 & 860 & 8 & 2 \\
\hline 18 & 270 & 240 & 20 & 10 \\
\hline 19 & 600 & 570 & 20 & 10 \\
\hline 24 & 1000 & 900 & 80 & 20 \\
\hline 25 & 700 & 600 & 70 & 30 \\
\hline 28 & 1100 & 1000 & 85 & 15 \\
\hline 29 & 2000 & 1950 & 30 & 20 \\
\hline 30 & 450 & 400 & 35 & 15 \\
\hline Total & 9490 & 8850 & 453 & 187 \\
\hline
\end{tabular}

Table 4 Abnormality detections are more in the COVID-19 period as shown for month May 2020 


\section{May-2020}

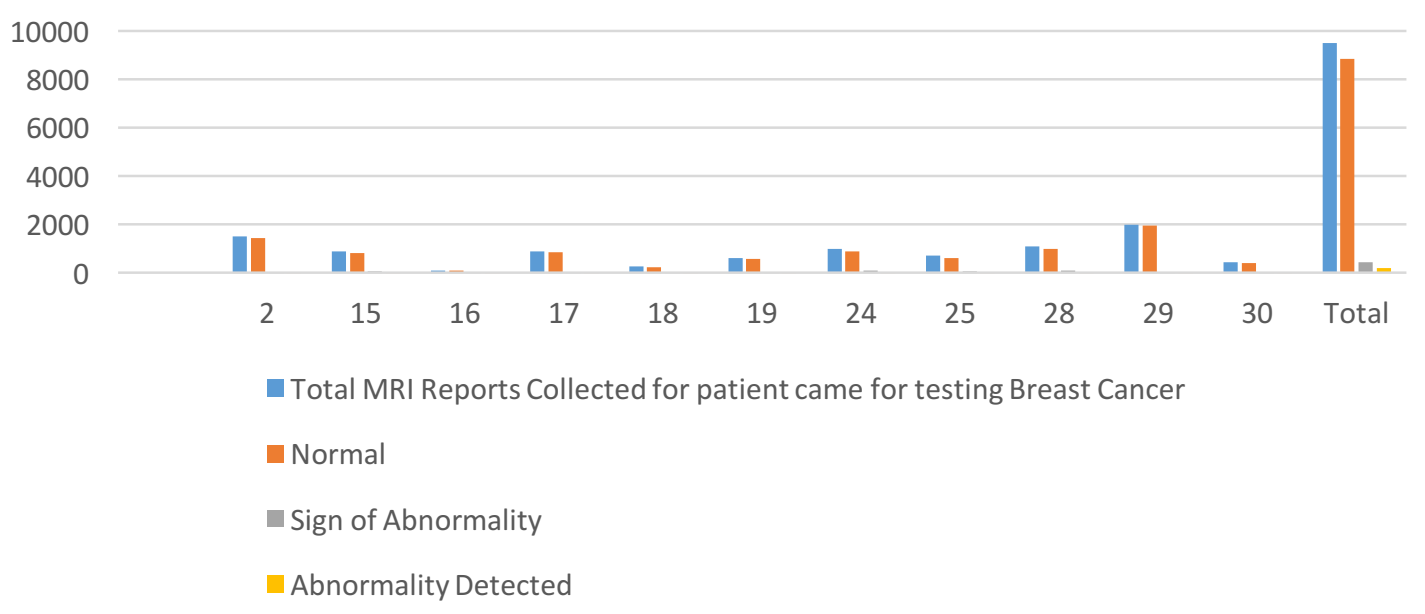

Figure 8 Sign of abnormality shows its seriousness

\begin{tabular}{|l|r|r|r|r|r|}
\hline Month & Total Collected MRIs & Accuracy & F1-Score & & Cohen-Kappa Score \\
\hline January & 551 & $83 \%$ & 0.79 & 0,21 \\
\hline February & 1718 & $82 \%$ & 0.88 & 0.29 \\
\hline April & 6297 & $89 \%$ & 0.91 & 0.35 \\
\hline May & 9490 & $80 \%$ & 0.93 & 0.33 \\
\hline Total & 18056 & & & \\
\hline
\end{tabular}

Table 5 Performance measure of the method is shown

\begin{tabular}{|l|r|}
\hline Month & Total Collected MRIs \\
\hline January & 551 \\
\hline February & 1718 \\
\hline April & 6297 \\
\hline May & 9490 \\
\hline Total & 18056 \\
\hline
\end{tabular}

Table 6 Total collected MRIs

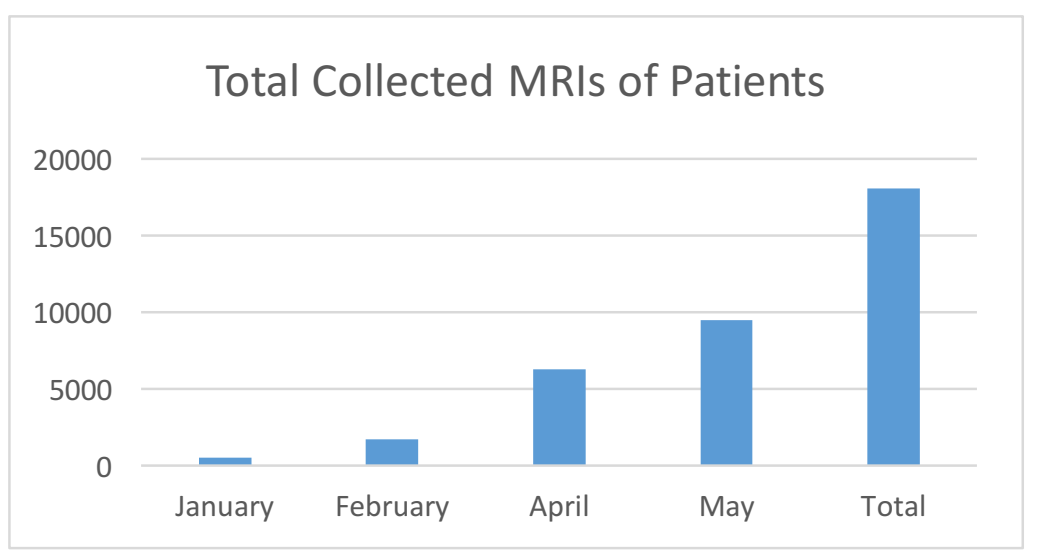

Figure 9 Graphical representation of collected data 


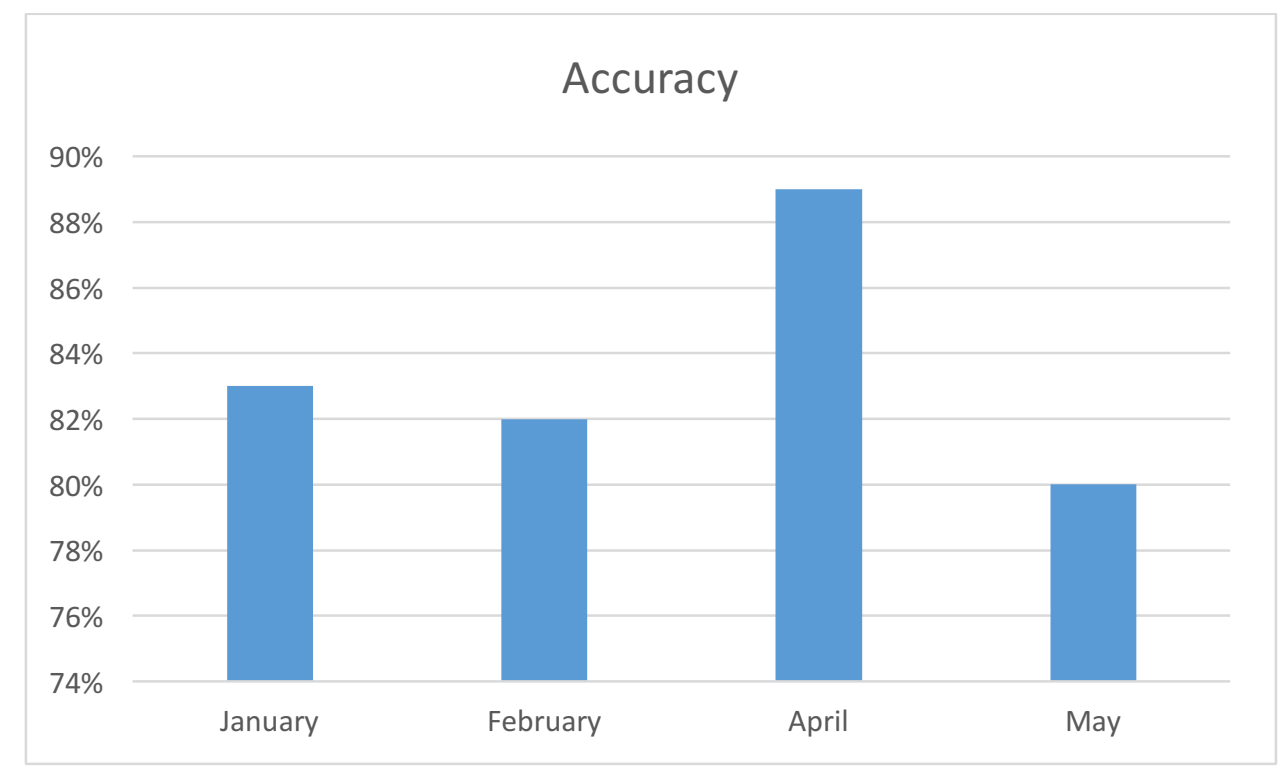

Figure 10 Accuracy measure before and after COVID-19

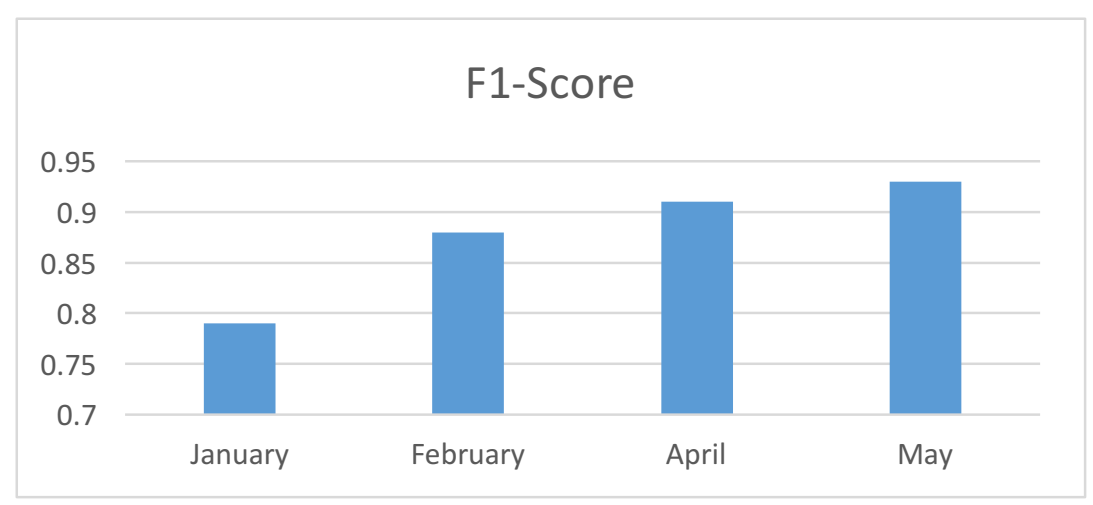

Figure $11 \mathrm{~F} 1$-score for four months

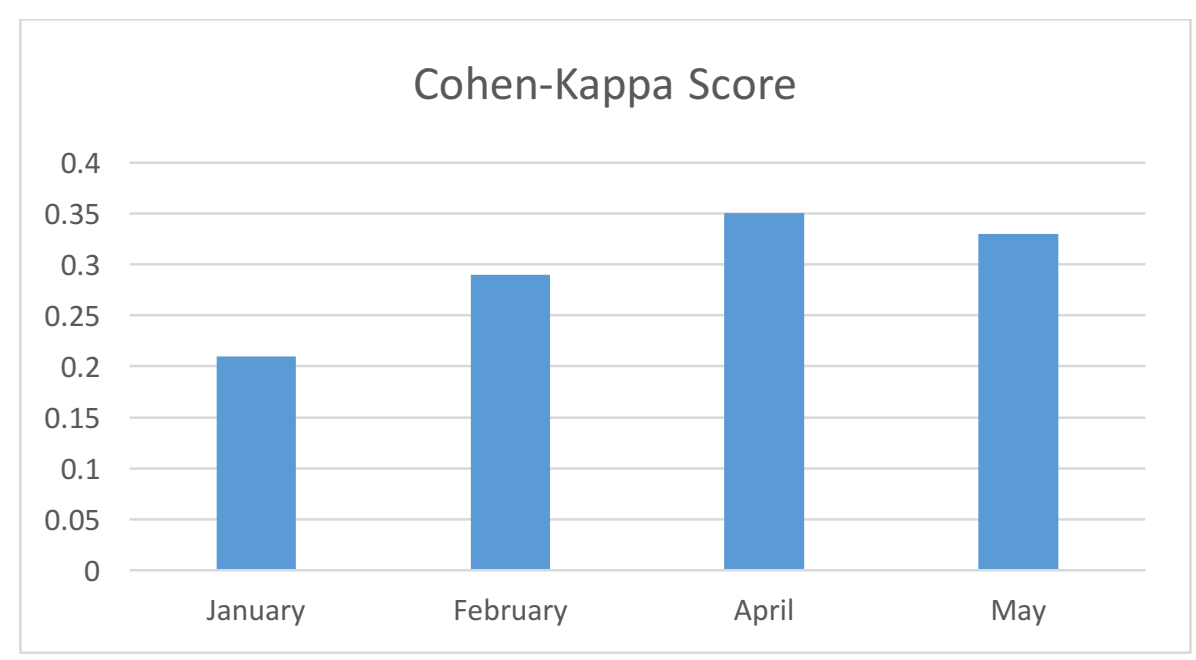

Figure 12 It shows the changes during COVID-19 


\section{Conclusions}

The datasets are collected from various hospitals in West Bengal. The results obtained by the proposed approach indicate that there is a variations of sign of abnormalities before and after COVID-19. It also correctly detected the abnormalities, if present. The performance measures such as Accuracy, F1-score and Cohen-Kappa score are calculated for four months and graphically presented in the result section.

\section{References}

1. American Cancer Society, Breast Cancer Facts and Figures 2019, American Cancer Society, Atlanta, GA, USA, 2019.

2. O. Ginsburg, F. Bray, M. P. Coleman et al., "The global burden of women's cancers: a grand challenge in global health," The Lancet, vol. 389, no. 10071, pp. 847-860, 2017.

3. E. B. Cole, E. D. Pisano, E. O. Kistner et al., "Diagnostic accuracy of digital mammography in patients with dense breasts who underwent problem-solving mammography: effects of image processing and lesion type," Radiology, vol. 226, pp. 153-160, 2003.

4. N. F. Boyd, H. Guo, L. J. Martin et al., "Mammographic density and the risk and detection of breast cancer," New England Journal of Medicine, vol. 356, no. 3, pp. $227-$ 236, 2007.

5. R. E. Bird, T. W. Wallace, and B. C. Yankaskas, "Analysis of cancers missed at screening mammography," Radiology, vol. 184, no. 3, pp. 613-617, 1992. 